TITLE ADAPTIVE DYNAMIC NETWORKS AS MODELS FOR THE IMMUNE SYSTEM AND AUTOCATALYTIC SETS

AUTHOR(S): J. Doyne Farmer, Stuart A. Kauffman, Norman H. Packard, and Alan S. Perelron

Conference Proceedings, "Perepectives in Theoret1cal Blology and Medicine, National Institute for Health, Bethesda, Maryland, held Apr11, 1986.

\title{
DISCLAIMER
}

This report wae proparyd as an acoount of work sponcored hy an asoncy of the United Statea Government. Nelther the Unitod Stutes Covernment nor eny asency thereof, not any of their omployeas, makes any warrenty, expreas of implled, of asaumes any legal liability or rosponalbility for the accuracy, completeneas, or urefulneas of any information, apparatua, product, or proceses dieclueed, or represents that lts use would not infringe privately owned rights. Raferonce heroin to any apocilio commercial product, process, or sorvice by trade name, trademark. manufacturer, or otherwise does not nocewarily conatitute or Imply its endorsement, recom. mendation, or favoring by the United State Government or any agency thereof. The viows and opintons of authon expreased heroin do not noceasurily stete or refloct thues of the United States Government or any agency thereof.

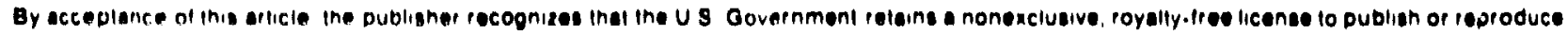
ine published lorm of inis contribution of 10 sllow others 10 do so. lor U 9 Oovernment durdoses

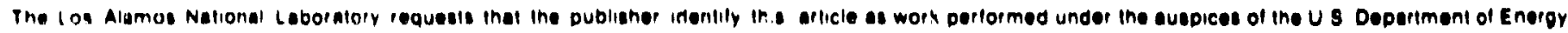




\title{
Adaptive Dynamic Networks as Models for the Immune System and Autocatalytic Sets
}

\author{
J. Doyne Farmer \\ Theoretical Division \\ Los Alamos National Laboratory \\ Los Alamos, NM 87545
}

Stuart A. Kauffman

Department of Biochemistry and Biophysics

School of Medicine, University of Pennsylvania

Philadelphia, Pennsylvania 19104

Norman H. Packard*

The Center for Nonlinear Studies

Los Alanos National Laboratory

Los Alamos, NM 87545

and

Alan S. Perelson

Theoretical Division

Los Alamos National Laboratory

Los Alamos, NM 87545

We describe a general class of network models that can be used to represent complex adaptive sysirms. These models have two purposes: On a practical level they are closely based on real bjological phenomena, and are intended to model detailed aspects of them. On a more general level, however, they provide a framework to address broader questions concerning evolution, adaptation, pattern recognition, and other properties of living systems. In this paper we concentrace on the more general level, illustrating the basic concepts with two examples, a model of the immune system and a model for the spontaneous emergence of autocatalytic sets in a chemically reactive polymer soup.

By its very nature adaptation involves the ability to change. We will distinguish two types of change, adjustment and innovation. An adjustment is a change that takes place through the alteration of an existing property, for example, a change in the size of a limb. An innovation, in contrast, is a change that cannot be made through the

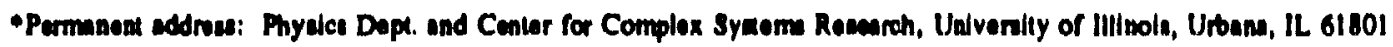


adjustment of an existing property, but instead requires the generation of something genuinely new. An example is antigenic shift in influenza virus; by recombination with another virus genes are gained or lost, generating a virus with significantly different properties from those of its predecessor. The significant aspect is not that the change is sudden, but that it results in qualitatively new features.

Traditionally, studies of adaptation have focused more on adjustment than on innovation. The reason for this, in part, stems from limitations in the common repertoire of dynamical models. In the usual dynanical model the state variables and parameters are specified at the outset. Although their values may change, the nature of the governing dynamical rule does not. This approach is naturally suited to modeling adaptations involving adjustment. The properties of the organisms or species in the system are represented by dynamical variables, with dynamical rules that take their interactions intu account. The standard tools of dynamical systems theory can then be used to analyze the behavior of the system as the variables and parameters adjust their values.

This approach does not work well, however, in modeling innovation. Once the variables and the dynamical framework are fixed there is no possibility to des rribe new phenomena that require additional state variables, precluding the emergence of any genuinely new features that might arise from such a change.

The adaptive dynamic networks that we discuss here are an method of modeling adaptation that allows the study of innovation as well as adjustment. The variables do not need to be specified in advance. Instead, each variable has a set of associated "properties". There is a rule that states how these properties interact to determine the equations of motion. This rule also determines how variables are spontaneously added or deleted as the system evolves. This in turn causes terms to appear or disappear in the equations of motion. New variables emerge automatically, with novel properties that are not necessarily specified in advance. Selection and adaptation occur spontaneously, much as they do in nature. The system evolves by changing its internal dynamics, with no need to impose an artificial construct, such as an arbitrary fitness function, in order to see adaptation take place.

To illustrate this approach we focus the discussion on two examples. One of these is a model for pattern recognition in the immune system ${ }^{1}$, and the other is a model for the catalytic replication of polymers ${ }^{2}$. Both of these models represent attempts to model the structure of real systems, incorporating their biological and chemical elements as ralistically as possible, within the constraint of tractability. In each case making an accurate model has naturally led us to formulate the problem in terms of an adaptive dynamic network. In the discussion here we concentrate on the highlights of the models, emphasizing the basic ideas rather than the detailed applications. We begin by discussing the autocatalytic replication of polymers, and then go on to discuss the immune sy'stem.

\section{Autocatalytic sets of polymers}

The adantive network described in reference 2 is a model for a soup of polymers. The soup is chemostat in which primitive building blocks such as amino acids are 
supplied, together with some form of energy to drive the system away from equilibrium. The building blocks form into more complicated moleculer by organizing themseives into one dimensional chains (polymers). The polymers may then catalyze reactions between other polymers in the system. Our goal is to demonstrate the formation of autocatalytic sets, i.e., sets in which every member is formed by at least one catalyzed reaction between other members of the set. We expect that autccatalytic sets can act as simple reproducing metabolisms, which can be viewed as prototypes for living organisms. There are several motivations for this study: (1) It is a model for the origin of life. (2) Aspects of these ideas may have potential industrial applications. (3) It provides a forum to study evolution. The idea to study autocatalytic sets in this context was originally proposed by Kauffman ${ }^{3}$.

The exact nature of the polymers is somewhat arbitrary, as long as they are one dimensional, and as long as they have catalyzed reactions of the type described below. When the model was originally formulated we had in mind polypeptides, which are known to have catalytic properties. However, any set of polymers with appropriate catalytic properties could easily be incorporated into our model. (For example, there is speculation that RNA-cacalyzed RNA replication may allow the formation of arbitrary RNA sequences ${ }^{4}$ ).

Since the polymers are one dimensional they can each be represented as a one dimensional string, with an alphabet whose letters represent the basic building hlocks. For example, if there are two building blocks $A$ and $B$, a polymer $a$ might be represented as $a=A B A A B B A B$. For polypeptides the letters would represent the basic amino acids (a 20 letter alphabet), or for RNA the leiters would represent nucleotides (a 4 letter alphabet).

We assume that the polymers have two types of chemical reactions, condensation or ind-joining reactions, and cleavage or splitting reactions. The reactions are reversible; a condensation can be reversed by the corresponding cleavage. Exchange reactions can be built out of combinations of condensation and cleavage. We assume that oul of all possible reactions there are some that are catalyzed in the presence of other polymers. Furthermore, we assume that the catalyzed reactions ai: so much faster than the uncatalyzed reactions that, at least to first approximation, the uncaialyzed reactions can be neglected. The basic reaction can be represented schematically as

$$
a+b \stackrel{e}{\rightleftarrows} c
$$

The polymer $c$ is assumed to be the concatenation of the polymers $a$ and $b$; for example, in a two letter alphabet where the basic building blocks are $A$ and $B$, if $a=A A A$ and $b=B B$, then $c=A A A B B$. $e$ is a polymer that catalyzes the reaction.

The difficult part of this problem from a physical point of view comes in assigning chemical properties to the polymers. For real molecules such as polypeptides, the chemical properties of given molecule are hard to predict from the chemical composition. There is in general no way to decide which molecules will act as catalysts for which reactions. We address this problem in our model in several different ways, the simplest of which is to select catalysts and reactions at random. Out of the set of all possible reactions, we randomly pick a fraction which we consider to be catalyzed and 
then randomly assign them reaction velocities in a physically reasonable range. In addition, we assign at random one of the polymers in the soup as the catalyst for that reaction. An alternate approach is to use a string matching procedure, as described in the discussion below of the immune system model. These two approaches represent two extremes; in the random model a change of one of the basic building blocks typically causes a total change in the chemical properties; whereas in the string match model it causes a smooth change. Real chemical reactions seem to fall somewhere in between.

The set of possible catalyzed reactions can be visualized as a graph, with arrows connecting the two cleavage products $a$ and $b$ and the condensate $c$ to a nodal point, corresponding to the reaction. The network of catalysts can be thought of as a siperimposed set of branches connecting the catalysts to the reaction nodes. (See FIGURE 1.) Assignment of chemical properties amounts to giving a procedure for determining the reaction and catalysis graphs, together with associated kinetic properties such as reaction velocities, equilibrium constants, etc.

To have interesting behavior we must pump energy into the system to drive if away from equilibrium. There are several possible ways to do this. One is to use mass action, and drive the system by simply providing a steady supply of simple molecules, which we call the food set. The food set might be just the basic bvilding blocks, $A$ and $B$, or it might include some short combinations of them as well. In a chemostat the volume of the system remains constant, so that as food is supplied to the system other material is washed out. Letting the reaction vessel overflow conserves volume and provides a simple form of energy dissipation. An alternative way to pump energy into the system is by energizing chemical bonds ${ }^{5}$. For polypeptide molecule;, for example, this can be done by attaching high energy phosphate groups.

Finally, in order to actually simulate this model we must specify the dynamics. The simplest case is to assume that the system is well-stirred, so that its behavior can be summarized in terms of the concentration of each polymer species. To model eyua. tion (1) accurately it is necessary to break it down into several intermediate reactions, involving each of the intermediate combinations that can be formed out of the catalyst and the products. This procedure complicates the simulation considerably. Instead, we have found an approximation that allows us to deal with saturation of the catalysts without udding equations for each intermediary complex ${ }^{2}$. As a result, we have a method of assigning to the reaction graph and its related properties a system of ordinary differential equations for the concentration of exsh polymer species.

As we stated in the introduction, an important property of this system is that the dynamics is not fixed. At any given time there is always a finite list of chemical species present in the soup, but as conditions in the system change, the list may change. The system thus explores potentially infinite space of possibilities, even though it remains finite as it does so.

The rule for deciding when to delete a species from the list is based on the notion that there is a minimum concentration threshold, corresponding to having one molecule present in the system. If the continuous concentration variable drops significantly below this threshold, the probability that this species is present is small, and it can ve 
eliminated from the system. Similarly, unless it is in the food set, a given species can be added to the system only if it is created by a reaction involving other elements already present. Its concentration is monitored, and when it acquires sufficient concentration to correspond to having at least one molecule it is added to the list of species actually in the system.

Thus, we must distinguish between the graph described above, which lists the possible reactions and species without taking the concentration into account, and the subset of this graph containing only the species whose concentrations are above the critical threshold. For convenience, call the graph described above the virtual graph, and the graph made including only those species above threshold the actual graph. The virtual graph is fixed by the rules of chemistry, whereas the actual graph depends on the species that are actually present, and is dynamic.

Our simulations thus far are in a preliminary stage. We have demonstrated that it is possible to make autocatalytic sets on the virtual graph, i.e., without taking the kinetics of the reactions into account. What remains to be done is to demonstrate that we can create autocatalytic sets in a realistic amount of time on the actual graph, i.e., taking the reaction kinetics into consideration. We are optimistic that this goa! can be achieved in the near future. Once this is done, we can begin doing evolutionary experiments, studying the competition for resources between different autocatalytic sets.

This model provides a potential bridge from simple molecules to the complex polymers and associated chemical pathways needed for contemporary life. The autocatalytic sets that we envisage can be viewed as prototypic life forms. The key difference relative to contemporary life forms is that they are a one-component rather than a two-component system. In contemporary organisms DNA contains the "blueprint" that allows an organism to be built, while proteins provide the catalytic machinery that allows this blueprint to be followed. The polymers in our model, in contrast, contain both at once. Their chemical properties determine their reactions with other polymers, which in turn determine whether or not they remain at positive concentration. Furthermore, this system can evolve; uncatalyzed reactions provide a background level of fluctuations which cause the creation of new species, which in turn has the potential to generate new autocatalytic sets. These sets compete with each other for raw materials, and those with the most favorable chemical properties are selev.ed. As the mixture becomes richer and more diverse, the rich chemical environment created could provide the raw materials and chemical pathways needed for the origin of contemporary life. Althoigh this scenario is currently most plausible for polypeptides, there are also possibilities that it could work for RNA molecules, or mixtures of RNA moleciles and proteins.

\section{Pattern Recognition in the Immune System}

Our second example of an adaptive system is the immune system. This system leams to recognize foreign organisms and exhibits the property of memory. It remembers that it has seen a foreign organism and on its second encounter with that organism it attacks it more rapidly and more efficiently. As we describe in more detail below, the functioning of the immune system depends heavily on innovation. Antibody molecules, 
which are used to recognize foreign :nolecules or antigens, are created by a seemingly random process. Those that are valuable to the system (in the sense defined later) grow in concentration, while those that are not die out. This basic process of pattern recognition and selection is known as clonal selection, and is similar to natural selection except that it occurs within the confines of the somatic cells of the immune system of a single organism. Adaptation occurs on a very rapid time scale, on the order of days or weeks.

The immune system is a set of cells and molecules. The molecules which we shall focus on here, antibodies, are analogous to the polymers in the autocatalytic network. Antibudies can be classified into types; each antibody of the same type has the same chemical structure. The set of all antibodies within an individual is very diverse and constantly changing. A given individual is thought to contain approximately $10^{7}$ to $10^{8}$ distinct antibody types. The total number of different antibody types that could be made is unknown, but is probably orders of magnitude higher than the set actually present in a given organism at any givelı time.

Antibodies recognize chemical features of antigens called epitopes. An antigen typically has more than one epitope, and a given antibody can typically recognize a whole range of antigens. The strength and specilicity of the interaction between the antibody and antigen is measured by the affinity of the interaction. The large repertoire of different antibody types, coupled with each antibody being able to interact with a set of different antigens, is thought to account for the ability of the body to recognize essentially any antigen ${ }^{6}$.

Antibodies are made by cells called $B$ lymphocytes. For each type of antibody there is a corresponding B lymphocyte, which produces only this type of antibody. During its differentiation in the bone-marrow each B cell expresses a single type of antibody molecule on its surface which serves as a receptor for antigen. When stimulated, the B lymphosyte proliferates and secretes its receptor molecule as a free antibody. Antibodies thus can either be free or receptors attached to cells.

Thinking of each different type of antibody as a species, we can represent the dynamics of the immune system as a set of differential equations for the concentration of each species. The number of diff, rential equations required to describe the system changes as novel species of antibody molecules are created, as useless species are eliminated, and as new antigens are encountered. As in the case of the autocatalytic network we can represent the possible interaction.s in the system in the form of a reaction graph. The main difference is that in its simplest form our nlodel has no analog of catalyzed reactions, since it considers only B lymphocytes. A more realistic model, however, must necessarily include another type of cells called $\mathrm{T}$ lymphocytes, which play a role similar to catalysts. (Helper T cells "catalyze" the production of antibodies by $B$ lymphocytes, while suppressor $T$ cells inhibit their production.)

One of the fundamental dogmes of immunoiogy is the theory of clomal selection. The basic idea is that the antibody molecules on the surface of $B$ lymphocytes are very diverse and to some extent randomly made. Many (possibly most) of the B cells have receptors that are useless and detect notining. However, by chance, some receptors have chemical properties that aliow them to react with antigen in the cell's environment. 
When this occurs the B cell is stimulated to grow into a clone of identical cells, each of which secretes free antibodies. In contrast, B cells that are not triggered live a few days and die. There is thus an amplification of both the cells and antibodies which are able to react with antigen. Free antibodies attach themselves to the antigen, flagging the antigen for removal by other components of the immune system. The net effect is a selection process whereby those antibodies that are useful in eliminating antigen are amplified, while those that are not die out. Because triggered $B$ cells live longer than $B$ cells that have never interacted with antigen the system maintains a memory of its exposures to antigen in the population levels of its different $B$ cell clones.

A central issue is regulation of the immune wponse. What determines the amplitude of the response, and how is it tumed off once the antigen is removed? Furthermore, how does the immune system prevent the amplification of self destructive antibody types? There are two basic ideas about how regulation is achieved. The simplest of these, based only on clonal selection, is that each antibody type is stimulated directly by antigen, and the immune response of each type occurs more or less independently of the other types. Thus there is no coupling between different B cell clones. Regulation of the immune response comes about through the action of helper and suppressor $T$ cells.

An altemative idea views the immune system as a regulated network of molecules and cells that recognize each other. Rather than having individual clones that respond to an antigen in isolation from one another, the immune system as a whole is involved in the response. The basic concept, due to Jerne ${ }^{7}$, is that because antibody molecules are created by random genetic mechanisms, they must look like novel molecules to the rest of the immune system and therefore must be treated like antigens. Thus the immune system will recognize its own antibodies as if they were foreign and make antibodies against them. Jerne suggested that during an immune response, antigen would directly elicit the production of a first set of antibody molecules, which he called $A b_{1}$. These then would act as antigens for other antibodies in the system and elicit their own immune response and cause the production of a second set of antibodies, $A b_{2}$, and so forth. Thus in an immune response antibodies respond to each other as well as to the antigen, and an entire $n$ twork of interacting cells and molecules is activated.

In the language of immunology the portion of an antibody that makes it distinct is called an idiotope. If a molecule has more than one idiotope, the set of all of them is said to define the molecule's idiotype. 'The network that Jerne envisioned has thus been called an idiotypic network.

Our model of the immune svstenı can be used to study both clonal selection and idiotypic networks. To assign chemical properties to antibody types, we represent each antibody by a string of ones and zeros. If we w/ant to study clonal selection this string will represent the untibody's paratope, which is the region of the antibody molecule that combines with antigen. If we want to include network interactions then we assume that each antibody, in addition to having a paratopi, also contains an epitope that other antibodies in the system can recognize. We thus enlarge our representation of an antibody to a pair of strings, one representing its paratope, and another 
representing its epitope. (See FIGURE 2.) If this epitope is unique, it will allow' the antibody to be distinguished from other antibody types, i.e., it will be an idiotope.

As in the autocatalytic polymer network model, we are forced to come up with a method of deciding which antibodies react with each other. The method that we have employed so far is complementary matching. For example, an antigen may have the string 11100011. An antibody with a paratope that had the complementary string 00011100 would "match" this antigen perfectly, and thus react with it strongly. Other antibodies which, say, only matched $90 \%$ of the bits in the antigen might also react with it, but not as strongly. According to the network theory, this string could instead represent the epitope of an antibody. Another antibody with a complementary paratope would attack it just as it would an antigen. For a description of how our model addresses clonal selection, see FIGURE 3.

Given a system of antigens and antibodies of specified "structure", i.e. with defined bit strings, it is an easy matter to determine who recognizes whom in the system. The recognition events can be coded into a graph with each node representing a species. A branch is placed between any two species which have more than a specinied degree of complementarity. The branch can be oriented so that the arrow points from a paratope to the epitope that it recognizes. The strength (i.e.affinity) of an interaction can be represented by assigning a real number to each branch, based on the number of complementary bits in the match. We call this number the "match strength".

A set of dynamical equations can be constructed once the composition of the network is known. Different levels of sophistication can be included in the dynamical model depending upon the goals one has for the model. For example, if we only wish to demonstrate that pattern recognition can occur in idiotypic networks, then simple dynamical laws based on predator-prey type equations will suffice. However, to model the various regulatory features of the immune system and concomitant phenomena such as low zone and high zone tolerance, then more complex equations are needed. Just to give the flavor of our approach, we examine a simple case. Consider a system containing $N$ antibody types, with concentrations $\left\{x_{1}, x_{2}, \ldots, x_{N}\right\}$, and $n$ antigens with concentrations $\left\{y_{1}, \cdots y_{n}\right\}$, and match strengths given by the matrix $m_{i j}$. The match specificities $m_{i j}$ take into account what reactions occur and how strongly. In defining $m_{i j}$ the convention is taken that the first index refers to the epitope, and the second to the paratope. The rate of change of antibody concentration is

$$
\dot{x}_{i}=c\left[\sum_{j=1}^{N} m_{j i} x_{i} x_{j}-k_{1} \sum_{j=1}^{N} m_{i j} x_{i} x_{j}+\sum_{j=1}^{n} m_{j i} x_{i} y_{j}\right]-k_{2} x_{i}
$$

We must also give an equation for the change in the concentration of the antigen $y_{i}$; the simplest case is that the antigen is neither growing nor decaying, except insofar as the immune system removes it. This implies that

$$
\dot{y}_{i}=-k_{3} \sum_{j=1}^{n} m_{j i} x_{j} y_{i}
$$

The first term in equation (2) represents the stimulation of the paratope of an antibody of type $i$ by the epitope of an antibody of type $j$. The second term represents the 
suppression of antibody of type $i$ when its epitope is recognized by the paratope of type $j$. The form of these terms is dictated by the fact that the probability of a collision between an antibody of type $i$ and an antibody of type $j$ is proportional to $x_{i} x_{j}$. The parameter $c$ is a rate constant that depends on the number of collisions per unit time and the rate of ancibody production stimulated by a collision. The constant $k_{1}$ represents a possible inequality between stimulation and suppression. This system is driven by the presence of antigens, represented by the third term. The final term models the tendency of cells to die in the absence of any interactions, at a rate determined by $k_{2}$. Equation (3) describes the elimination of antigen from the system. $k_{3}$ is an arbitrary rate constant.

As the concentration $x_{i}$ changes, the list of variables present in the system can change. These changes vccur much as they do in the autocatalytic reaction network. If the concentration of any antibody or antigen reaches a lower threshold level we assume the last molecule of that type has been eliminated, and remove that species from the system. Inputs to the system come both from new antigens and new antibody types. When the list of variables $x_{i}$ and $y_{i}$ change, there is an induced change in the matrix $m_{i j}$, altering the dynamics in equations (2) and (3).

In the real immune system, new antibody types are generated by several different mechanisms. First, by complex gene shuffling mechanisms, diverse types of new lymphocytes are created in the bone marrow. Further diversity is introduced through mutation of stimulated lymphocytes. In fact, the genes which determine the type of antibody a lymphocyte makes mutate at a rate that is much higher than that of other genes. All of these processes are easily taken into account in our model.

As a whole, the system is adaptive. Its operation ensures that antibodies that recognize things are amplified and antibodies that are inert are eliminated. Antigen which is recognized by antibodies is also eliminated. By having a source of new, novel antibodies the system learns to cope with unexpected antigens. The only way an antigen can win is to grow faster than the immune system can kill it, avoid detection altogether by hiding or by being so novel that nothing in the system recognizes it, or as in the case of AIDs, attack the immune system itself and prevent it from operating correctly.

As an adaptive system, what does the idiocypic network provide over simple clonal selection? This is an important question that our simulations are just beginning to answer. First, if an antigen has many epitopes (as is the case for any common pathogen), the system could learn that the occurrence of one epitope is correlated with the occurrence of others. Thus, by "network interactions" an alarm could be raised through out the system so that clones of many different specificities all get excited and start secreting antibody. This phenomena would be reminiscent of associative memory in the brain ${ }^{8}$. Second, as already pointed out by Jerne, each epitope could have an "internal image" within the immune system. For example, such an image could correspond to an anti-idiotypic antibody whose paratope was complementary to the paratope of an antibody that recognizes the antigen. The antigen could then be eliminated from the system, but its memory could be carried by network interactions involving the anti-idiotypic antibody. Thus the system would have elements in high 
concentration that would recognize the antigen if it ever appeared again, because it had elements interacting with its internsl image. Third, it has been hoped that the network vould provide a way of regulating the immune system. A clear picture of this has not yet been worked out. Horwever, if one thinks of the idiotope as providing a tag for subsets of antibody molecules or cells, the potential exists for independently directing the activities of many subpopulations of molecules and cells.

When Jerne first proposed the idiotypic network he suggested that the system operates at a steady state in the absence of antigen; antigen simply causes a perturbation away from this steady state. Upon termination of the response to the antigen the system would then return to a steady state, either its original one, or in the case of memory, suppression, etc, to a new steady state. Our simulations of the immune network provide a cifferent, more dynamic view. At a given time, some parts of the immune system may be oscillating, while other parts may be inhibited or stimulated. We hypothesize that the information about the immune system's history, for example, its exposure to a partizular epitope, may be stored in the dynamics of loops and more complex topoiogical structures of interacting cells and molecules. Thus there is a pattern of reactivity within the system and a set of connections between elements that is constantly changing as the system is exposed to antigen, as new antibody types are introduced from the bone marrow, and as existing types mutate and die. The pattern is something like a weather pattern: It constantly changes, never going to a steady state, though there are distinct periods of one type of behavior or another. Encounter with antigen is one type of behavior, but so is the natural internal activity of the system. For a system with as many elements as the immune system, i.e. $10^{8}$, it may be difficult to tell normal periods of activity from immune responses unless they are of the extreme type created in the laboratory. Our adaptive network simulations may give us a port hole from which to view the natural behaviors of a large dynamic, evolving immune system.

\section{Conclusions}

The adaptive dynamir networks discussed here provide a means to model aspects of adaptation including processes of innovation as well as adjustment. Unlike conventional dynamical models, the number of variables is not fixed, but instead changes in time as conditions within the system change. To accomplish this, variables are assigned properties which make them distinct from each other; given a list of variables and their properties, there is an algorithm that determines their interactions at any given time, and also determines when and how to introduce new variables. This approach is one level more flexible than that of conventional dynamical models; rather than fixing the number of variables and the dynamical rule, we fix the algorithm that generates them. This algorithm can be viewed as a kind of "metadynamics".

Our approach should be contrasted to the conventional dynamical systems approach. For example, to represent the autocatalytic network using fixed dynamics requires an infinite number of continuous variables. Even if initially only a finite number of these variables have nonzero concentration, subsequent evolution arcording to the law of mass action will cause all of them to acquire some nonzero concentration, albeit very small. Since there is no way to know in advance which variables are 
actually important, there is no way to restrict the dynamics to finite dimensions. With our approach, however, we discover which variables are important as the system evolves. The key to achieving this is the concentration threshold. The system is finite aimensional at all times, even though it potentially wanders through an infinite dimensional space. The fact that the system is finite at any given time means that we can actually simulate it on a computer.

In the two models that we discuss here the algorithm generating the adaptive network emerges naturally from modeling biological problems. In general this approach could also be used to model other types of systems. For example, it could be applied to problems in game theory; the properties of the variables could represent strategies their values a score of their usefulness. In fact, an approach that is analogous in many respects has been employed by Holland ${ }^{9}$, in his classifier model for machine learning. (For a review of this connection see reference 1.)

Our approach provides a sharp contrast to other approaches to understanding adaptation. For example, in the Fisher-Wright model organisms are assigned a "fitness function", which determines their likelihood to reproduce in the next generation. This function lives over an abstract domain whose axes are the characteristics of the organisms. When they breed their progeny acquire new characieristics, which in general also changes their fitness. This model is used as an abstract tool to deduce qualitative aspects of evolution.

The problem with this approach is that we know very little about the fitness functions of real organisms. They must certainly be very complicated, but there is no way to determine even in a qualitative sense just how complicated. (Are they fractal, for example?) Another problem is that in reality the fitness of a given organism depends on interactions with other organisms. As the other organisms change through evolution, so does fitness. Thus in reality fitness is always in flux, depending on the characteristics of all the organisms present. Hown the fitness landscape is influenced by taking this into account is a matter of pure speculation. The problems with the fitness function approach have been emphasized by Conrad ${ }^{10}$.

In contrast, for the adaptive netwo:k models discussed here there is no need to specify a fitness function by hand. Fitness is implicit in the system, and evolves in a natural way as the dynamical variables change their values and identities. The abstraction is thus brought down a level; rather than specirying a fitness function, we specify an algorithm for changing the list of variables and determining their relation to each other. Here we have the advantage that we can use the qualitative properties of reai systems as a guide. Furthermore, we; can try different algorithms to check the robustness of the properties that we observe. Ultimately, we hope that adaptive dynamic networks will provide a tool for understanding problems of adaptation in many different contexts. 
1. Farmer, J.D., Packard, N.H. \& A.S. Perelson. 1986. The immune system, adaptation, and machine learning. Physica 22D (in press).

2. Farmer, J.D., Kauffman, S., \& N.H. Packard. 1986. Autocatalytic replication of polymers. Physica 22D (in press).

3. Kauffman, S. 1986. Autocatalytic sets of proteins. J. Theoret. Bio. 119: 1.

4. Cech, T.R. 1986. A model for the RNA-catalyzed replication of RNA. Proc. Nat. Acad. Sci. 83: 4360.

5. Fox, R.F. 1982. Biological Energy Transduction: The Uroboros. WileyInterscience. New York.

6. Perelson, A. S. \& G. F. Oster. 1979. Theoretical studies of clonal selection: Minimal antibody repertoire size and reliability of self-non-self discrimination. $J$. Theoret. Biol. 81, 645-670.

7. Jerne, N.K. 1974. Toward a network theory of the immune system. Ann. Immunol. (Inst. Pasteur) 125C: 373.

8. Kohonen, T. 1977. Associative Memory: A System Theoretic Approach. Springer Verlag. Berlin.

9. Holland, J.H. Escaping brittleness: The possibilities of general purpose learning algorithms applicd to parallel rule base systems. Machine Leaming 2, chap. 26. Morgan Kauffman. Los Altos.

10. Rizki, M.M. \& M. Conrad. 1986. Computing the theory of evolution. Physica 22D (to appear). 


\section{Figure Captions}

1. A typical example of a graph that might describe an autocatalytic set. The reactions are represented by nodes connecting cleavage products with the corresponding condensate. Dotted lines indicate catalysis pathways, and point from the catalyst to the reaction being catalyzed.

2. A schematic representation of the structure of an antibody, an antibody as we represent it in our model, and a B-lymphocyte with antibodies on its surface that act as antigen detectors.

3. A schematic illustration of clonal selection. At the top, a foreign molecule (i.e. an antigen) is represented as having 3 epitopes, each represented by the binary string 11100111. The bone marrow, shown on the left, pumps a diverse set of Blymphocytes into the system, each of which has a large number of identical antibodies attached to it thi.t serve as receptors. We show five different antibody types, labeled $63,2,19,7$, and 14i, each of whose antibody types have a unique shape. Lymphocytes of type 2 have receptors with a paratope 00011000 which is perfectly complementary to the epitope of the antigen, indicating a strong reaction. Lymphocytes of type 7 have receptors that are partially complementary to the epitope (matching in 6 of 8 positions), indicating a weak reaction, and the remaining types match so poorly that they effectively do not match at all, and therefore do not react. Upon exposure to the antigen lymphocytes of type 2 and 7 are stimulated to proliferate. They also differentiate into a state in which they secrete a massive amount of free antibodies. The degree of stimulation and hence the final quantity of antibody secreted is proportional to the degree of matching between the receptor and the antigen, so that lymphocytes of type 2 are strongly stimulated, whereas lymphocytes of type 7 are only weakly stimulated. L.ymphocytes of types 63,19 , and 141 are not stimulated at all, and eventually die and leave the system. 

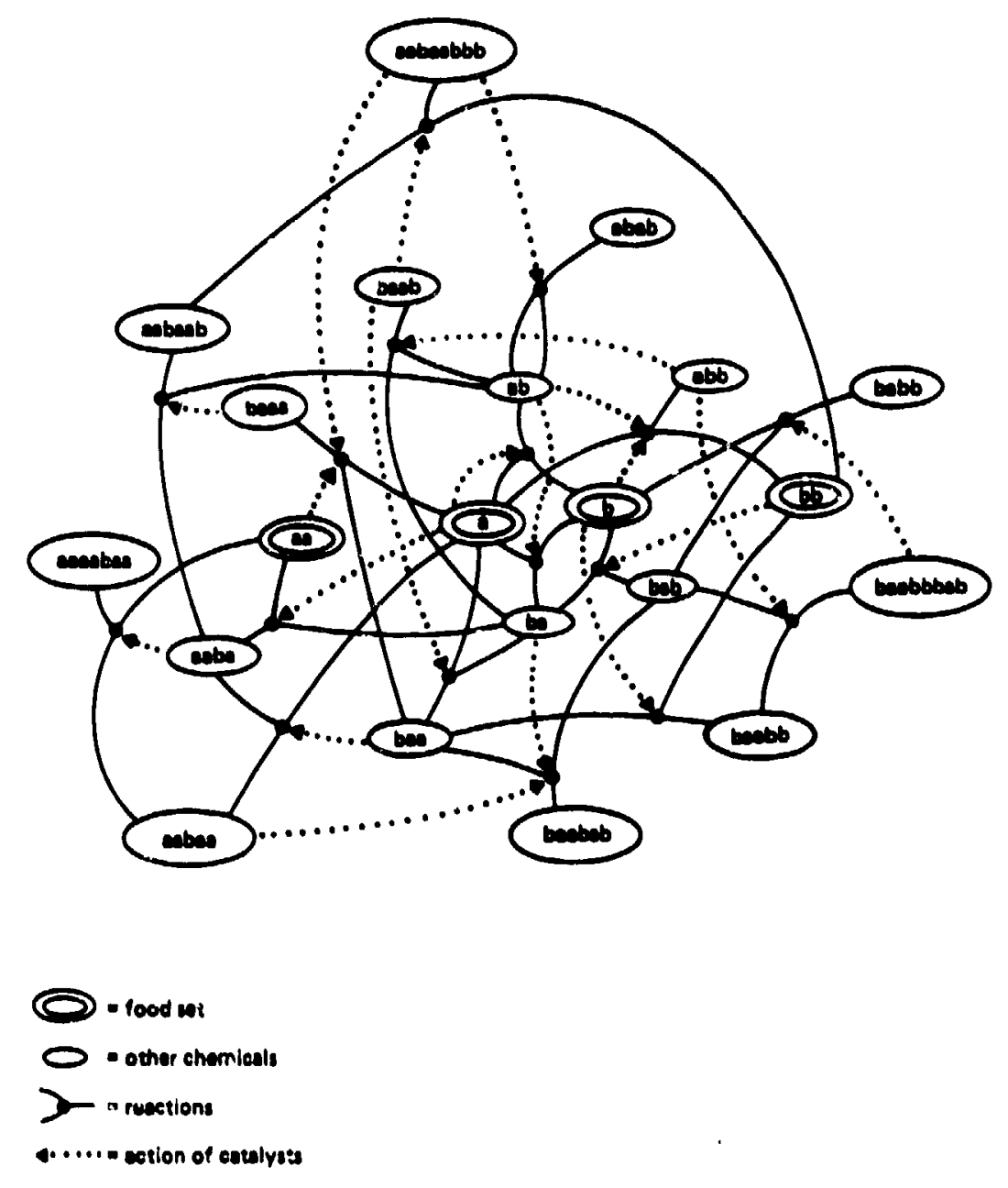

Farmer et. al.

Figs. 1.

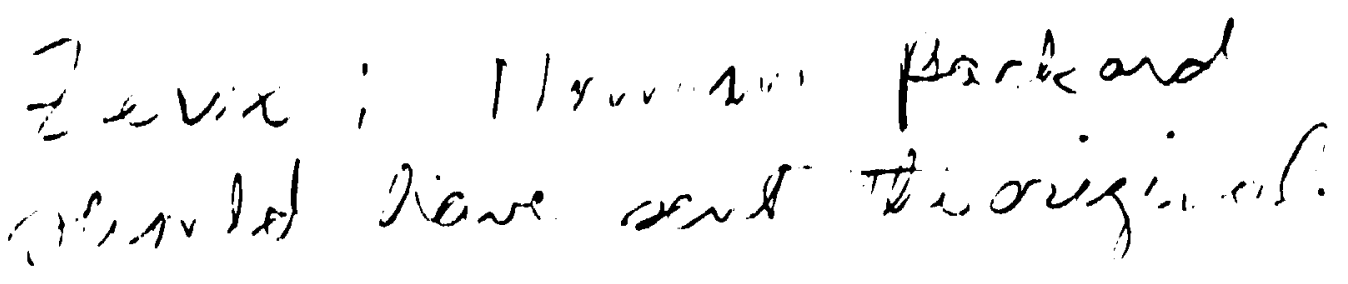




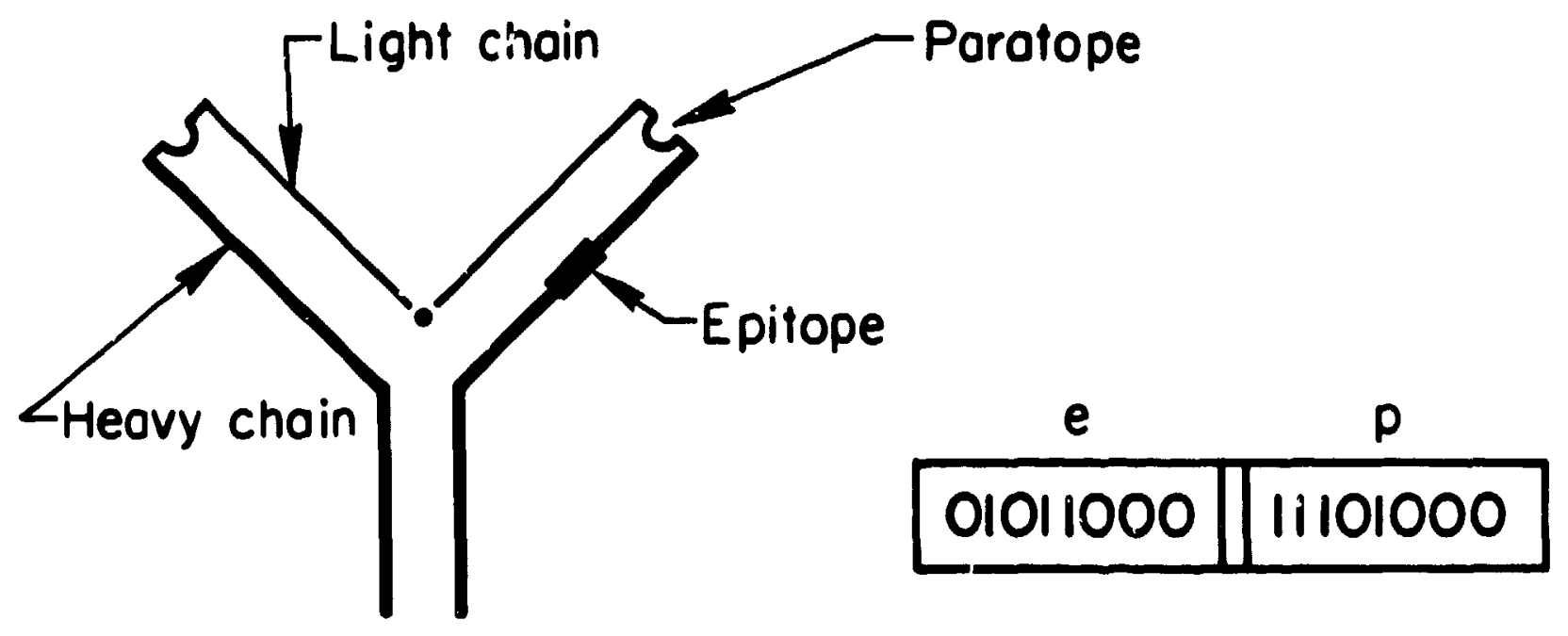

Antibody

Antibody Representation

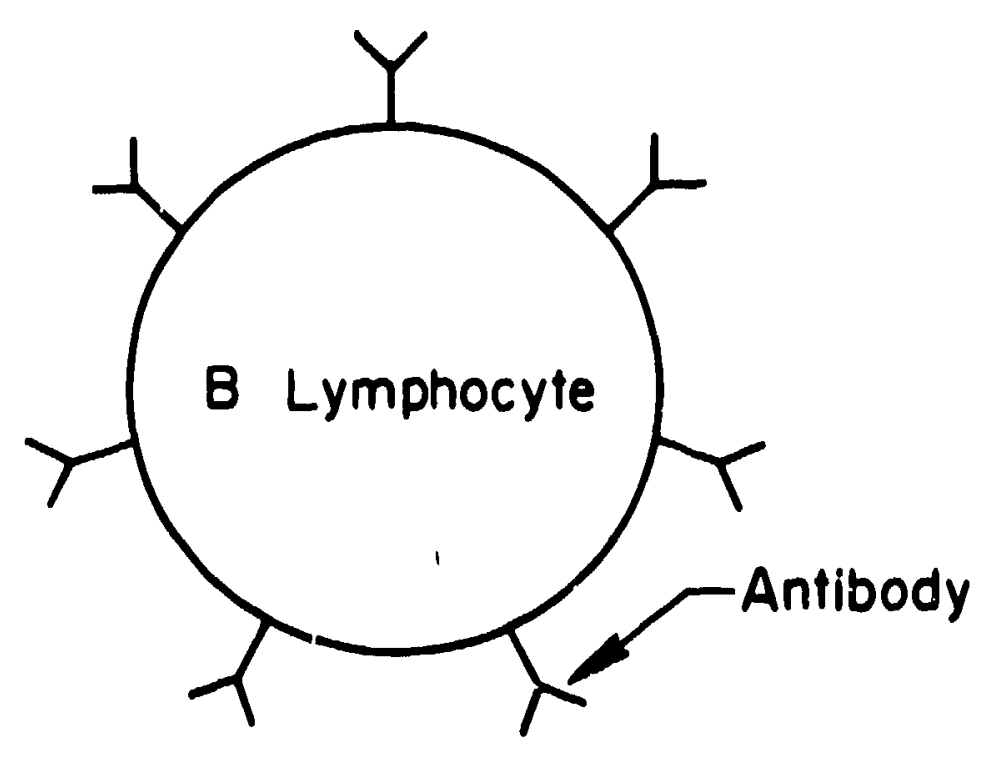




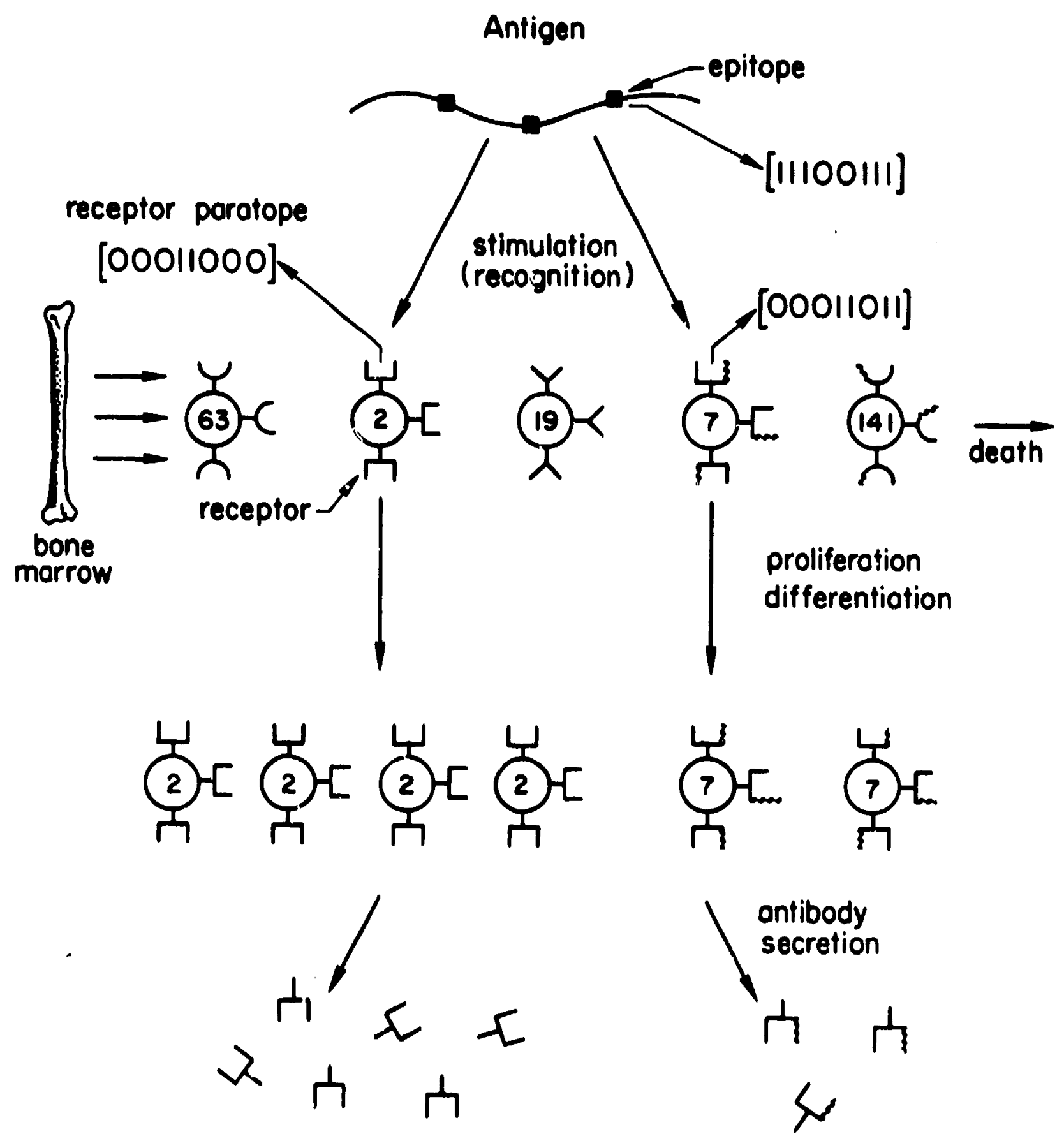

